\title{
Is Kyphoplasty Necessary?
}

\author{
Shigeo Ishiguro ${ }^{1}$, Koji Akeda ${ }^{2}$, Masaya Tsujiii ${ }^{2}$, Akihiro Sudo ${ }^{2}$ \\ ${ }^{1}$ Department of Orthopaedic Surgery, Sakakibara Spa Hospital, Tsu, Japan \\ ${ }^{2}$ Department of Orthopaedic Surgery, Mie Universtiy Graduate School of Medicine, Tsu, Japan
}

\begin{abstract}
We describe four cases of delayed union in female patients with severe osteoporotic vertebral fractures, which were treated in a similar but less costly method to kyphoplasty. Due to domestic regulations, inflatable tamps for kyphoplasty are not available to every clinical orthopedists in Japan. In our clinical experience of four cases of delayed lumbar spine union between 2009 and 2010 , we performed vertebroplasty using a reduction and spreading prod (Oyamada prod) for fracture reduction and a pediatric uromatic balloon (Medicon Co. Ltd.) to enlarge the pre-existing cavity. Our clinical results were comparable to those of kyphoplasty procedures performed in the USA. Our procedure could be used to overcome the shortage of medical supplies in developing countries or in countries such as Japan, which often prioritize financial concerns over providing optimal health care. Our method could serve as a useful compromise for moribund patients considering its cost efficiency.
\end{abstract}

Keywords: Osteoporotic vertebral fracture; Kyphoplasty; Pediatric uromatic balloon; Cost-efficiency

\section{Introduction}

The efficacy of kyphoplasty developed in the USA has been demonstrated since 2000, and it has contributed to immediate pain relief, less morbidity, and shorter hospital stays. However, as spiraling medical costs are a big concern in the USA, a less expensive method of using a kyphoplasty device was recently tested in a clinical trial [1]. Inflatable tamps for kyphoplasty are not always available to every orthopedic surgeon in other countries due to domestic regulations or poor economic conditions, which is true in Japan.

\section{Technical Notes}

In our clinical experience of four cases of delayed lumbar spine union between 2009 and 2010, we performed vertebroplasty using a reduction and spreading prod (Oyamada prod, Pentax Fujitoku Precision Corp., Tokyo, Japan) for fracture reduction and a pediatric uromatic balloon (Medicon Co. Ltd., Osaka, Japan) to enlarge the preexisting cavity $[2,3]$.

Two months had already passed when the patients first visited us following missed diagnoses and poorly performed conservative treatments. The patient ages at the time of the operations were $70,79,80,81$ years.

The surgical procedure is described with an illustrative case of a 70-year-old female. Patients were placed in the prone position on a Jackson table under general anesthesia. Oyamada prods were introduced through bilateral pedicles through a very small longitudinal skin stab incision on the bilateral pedicle under a C-arm confirmation, using the cannulated drilling system and dilation technique. This technique helped further enlarge the fracture cavity and reduce the vertebral body (Fig. 1). One $\mathrm{mL}$ syringes ( $8 \mathrm{~cm}$ in length), were cut to approximately $6 \mathrm{~cm}$

Received Jun 16, 2012; Revised Aug 6, 2012; Accepted Aug 13, 2012

Corresponding author: Shigeo Ishiguro

Department of Orthopaedic Surgery, Sakakibara Spa Hospital,

Sakakibara-cho, Tsu 1033-4, Mie prefecture 514-1293, Japan

Tel: +81-59-252-1111, Fax: +81-59-252-0522, E-mail: stoneblack1496@syutaikai.jp 
(JMS Co. Ltd., Tokyo, Japan) and set into both sides of the pedicle. The vertebral body was irrigated with saline, which was injected into one of the pedicles. The return stream was allowed to exit on the contralateral pedicle (Fig. 2). Saline was also irrigated from the opposite side of the pedicle. We routinely removed any sequestered hematomas using saline. Fig. 3 shows the lateral radiographs in extension and flexion.

A pediatric uromatic balloon (size $8 \mathrm{Fr}$, Medicon Co. Ltd), whose diameter before inflation was $2.7 \mathrm{~mm}$ and length was $30 \mathrm{~cm}$, was introduced into the cavity and inflated using contrast medium (Fig. 4). Balloon inflation further enlarged the pre-existing fracture cavity and made it more apparent (Fig. 4).

Approximately 3 to $5 \mathrm{~mL}$ of contrast medium was required to inflate the balloon fully to create the cavity.

Obstructing one side of the pedicle using a finger made it impossible to inject saline from the other side. The cavity was then considered to be closed except for the bilateral pedicle route. If it was possible to inject saline from the other side to that of the pedicle obstructed by a finger, we checked where the contrast medium was leaking. We used an ordinary $5 \mathrm{~mL}$ syringe and nelaton catheter (Terumo Co., Tokyo, Japan) to inject polymethylmetacrylate (PMMA) into the vertebral body. In cases of confirmed contrast medium leakage into the spinal canal, the PMMA was carefully injected using guidance from an image intensifier. PMMA was introduced from the anterior to the posterior wall, and the PMMA injection was stopped the moment the PMMA reached the posterior wall. Fig. 5 shows the postoperative radiographs of an illustrative case, and Fig. 6 is the 1-year postoperative computed tomography scan.

The kyphosis index (anterior wall height/posterior wall

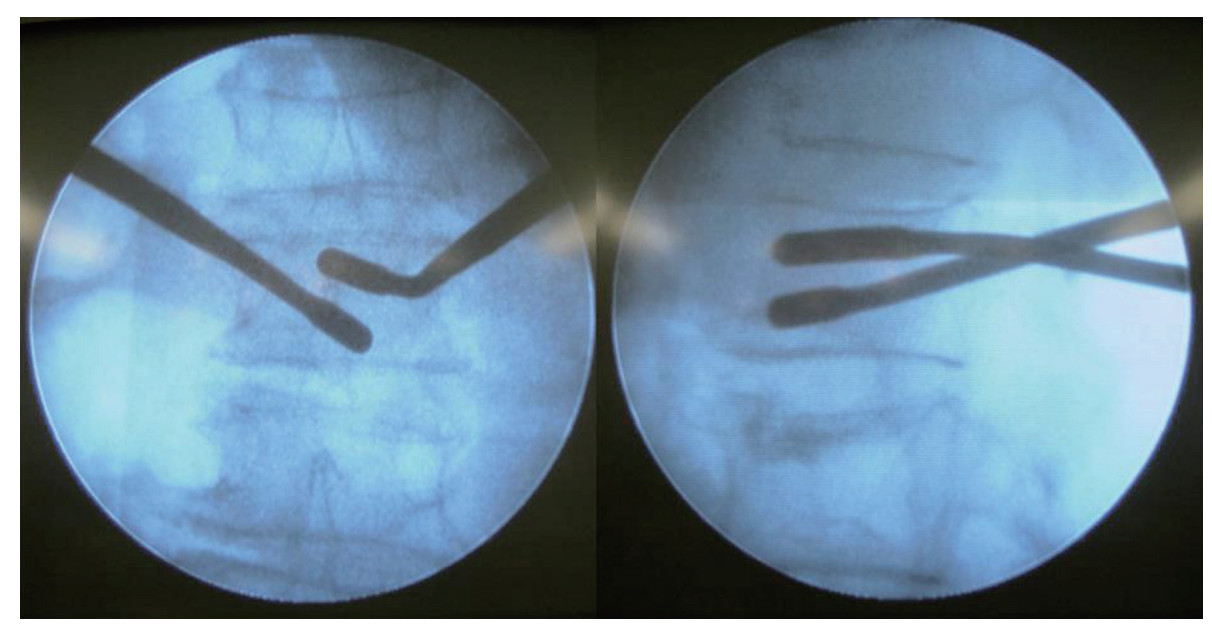

Fig. 1. Oyamada prods introduction through bilateral pedicles.

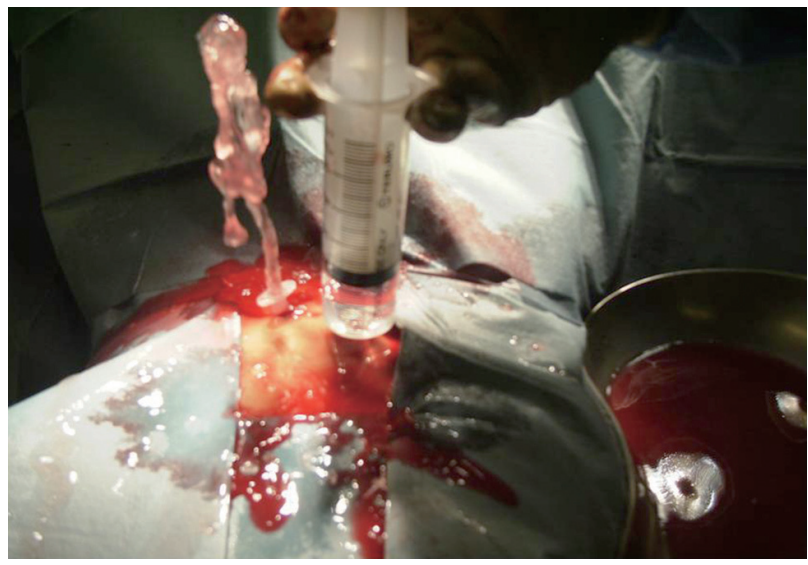

Fig. 2. The vertebral body was irrigated by saline, which was injected into one of the pedicles. The return stream was allowed to exit on the contralateral pedicle.

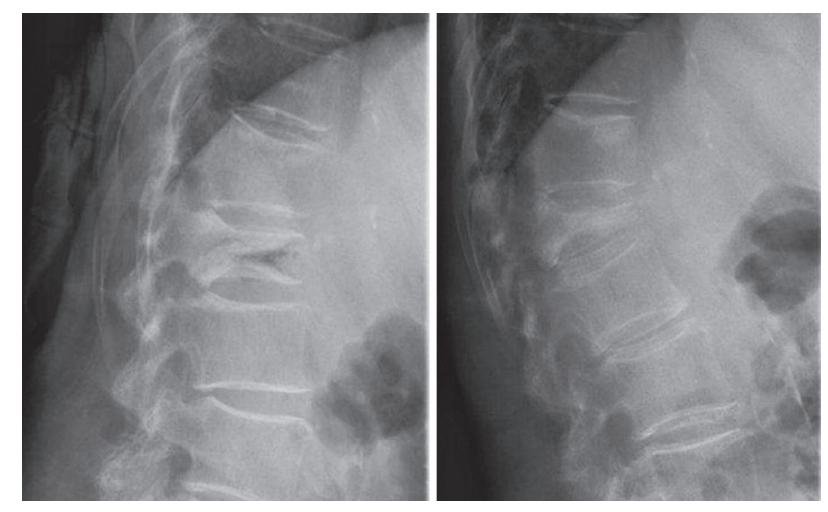

Fig. 3. The lateral radiographs in extension and flexion. 
height $\times 100$ ) improved from $15,69,65,75$ to $82,100,95$, 85. Visual analogue scale readings decreased from $9,8,7$, 8 to $2,1,2,3,1$ day after the operation, and patients could walk immediately after surgery. Minor cement extravasation occurred outside of the vertebral body in one female, with no clinical sequelae. No adjacent fractures were observed at the 1-year follow-up, and patients had returned to their previous daily living activities.

\section{Discussion}

A definite advantage of our method over kyphoplasty is the use of cheap, disposable devices such as inflatable balloon tamps, and the use of re-useable devices such as the Oyamada prod. In fact, the total price of disposable items for our method was 300 yen, whereas an inflatable balloon tamp alone sells for 390,000 yen in Japan. The cost of the inflatable balloon tamp is so high that a surgeon's fee for single level kyphoplasty was not profitable for the hospital unless the procedure was combined with other operations.

We have performed the same operative procedure using prods in the past (made from 3-mm or 4-mm Steinmann pins), and filled the fracture cavity with calcium phosphate cement [4]. Some Japanese authors have described a surgical method of introducing urinary balloons into the vertebral fracture site to enlarge the preexisting fracture cavity $[2,3]$. We suggest that a mixture of the two different techniques reduces medical costs, provides economic benefits to the medical institution, and secures comparative clinical results to those of kyphoplasty.

In addition, a possible theoretical advantage of our method over kyphoplasty is mechanical strength. An inter-commutable channel was formed between both pedicles at the anterior part of fractured vertebral body. PMMA was spread along the channel to make one big nugget of cement. This large cement mass provided more
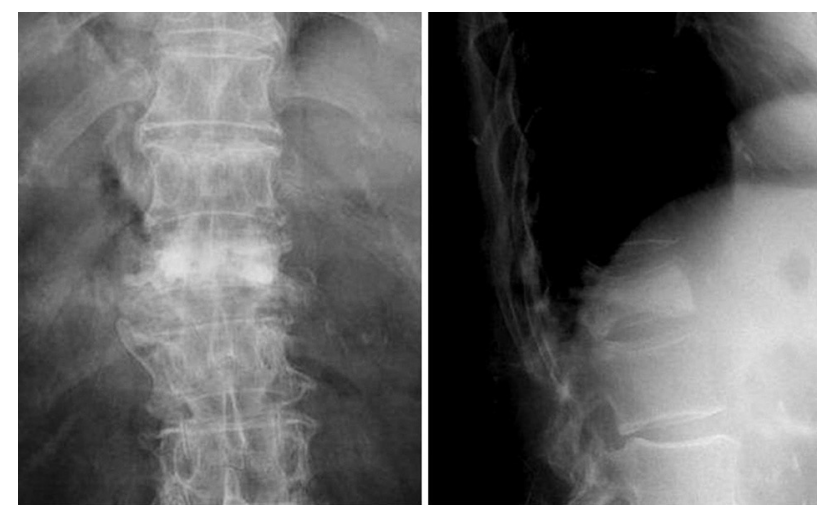

Fig. 5. Postoperative radiological radiographs.

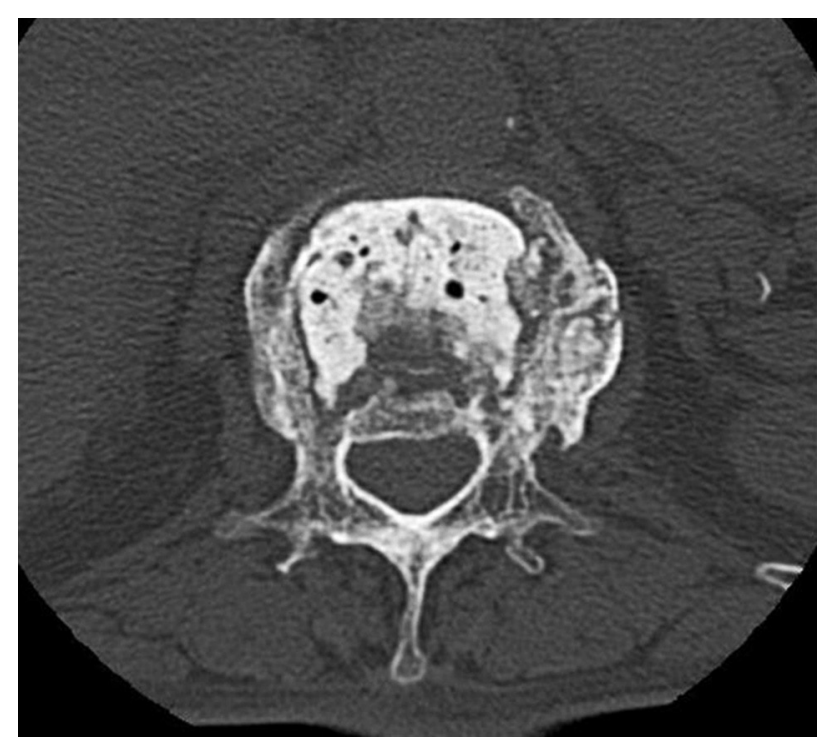

Fig. 6. The 1-year postoperative computed tomography.

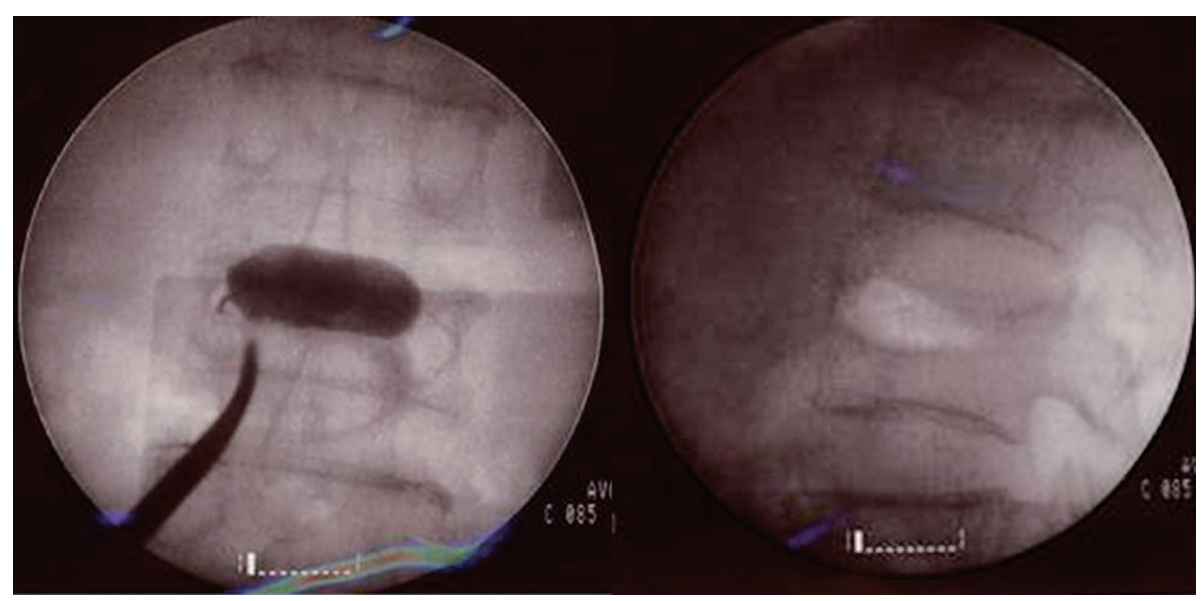

Fig. 4. The cavity enlargement by a pediatric uromatic ballon. 
biomechanical strength than that of kyphoplasty. However, there may be increased chance for complicating adjacent vertebral fractures compared to that of kyphoplasty, as our method provides a more rigid and strong mass. We suggest that one large cement mass would have a slim chance of being displaced either into the spinal canal or outside the vertebral body, even if an adjacent fracture actually develops.

Our method requires more careful technique than that of routine kyphoplasty, but adept skill and effortless endeavor could supersede the use of expensive devices. This method works to overcome shortages of medical supplies in developing countries or in countries such as Japan, which have a tendency to place financial concerns above a desire to provide optimal health care.

Our clinical results were comparable to those of previous studies $[1,5]$, and we believe that our method can serve as a useful compromise for moribund patients.

\section{Conflict of Interest}

No potential conflict of interest relevant to this article was reported.

\section{References}

1. Papadopoulos EC, Edobor-Osula F, Gardner MJ, Shindle MK, Lane JM. Unipedicular balloon kyphoplasty for the treatment of osteoporotic vertebral compression fractures: early results. J Spinal Disord Tech 2008;21:589-96.

2. Hoshino M, Nakamura H, Konishi S, et al. Endoscopic vertebroplasty for the treatment of chronic vertebral compression fracture: technical note. J Neurosurg Spine 2006;5:461-7.

3. Ishiguro S, Tsujii M, Sudo A. Successful bone union following calcium phosphate cement-assisted percutaneous transpedicular balloon kyphoplasty of a large interbody cleft on long-term hemodialysis patient. Asian Spine J 2011;5:188-91.

4. Ishiguro S, Kasai Y, Sudo A, Iida K, Uchida A. Percutaneous vertebroplasty for osteoporotic compression fractures using calcium phosphate cement. J Orthop Surg (Hong Kong) 2010;18:346-51.

5. Kim KH, Kuh SU, Chin DK, et al. Kyphoplasty versus vertebroplasty: restoration of vertebral body height and correction of kyphotic deformity with special attention to the shape of the fractured vertebrae. J Spinal Disord Tech 2012;25:338-44. 